\title{
Leishmaniose tegumentar na Região Metropolitana de Belo Horizonte: aspectos clínicos, laboratoriais, terapêuticos e evolutivos (1989-1995)
}

\author{
Cutaneous leishmaniasis in the Metropolitan Region of Belo Horizonte: \\ clinical, laboratorial, therapeutic and prospective aspects
}

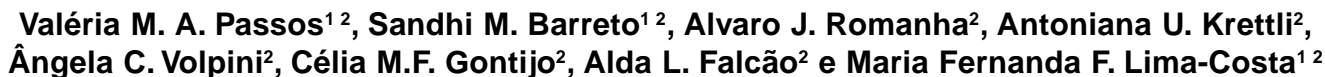

Resumo Foram investigados aspectos clínicos, laboratoriais, terapêuticos e evolutivos da leishmaniose tegumentar americana em Belo Horizonte. O estudo incluiu 358 pacientes com leishmaniose cutânea (LC) $e$ $25 \mathrm{com}$ leishmaniose mucosa (LM). Comparados aos pacientes com LC, aqueles com LM apresentaram maior tempo de doença e relato de outras doenças concomitantes, sugerindo que a debilitação pela leishmaniose e/ou outras doenças podem contribuir para a ativação e/ou disseminação mucosa do parasito. As sensibilidades das reacões intradérmica, de imunofluorescência indireta e da pesquisa direta do parasito foram de 78,4, 79,3 e 68,3\%, respectivamente. O tratamento com antimoniato de meglumina foi $100 \%$ eficaz, com 59\% de efeitos colaterais ao longo do tratamento. A recidiva após tratamento ocorreu em $32(10,1 \%)$ dos 318 casos seguidos por até dois anos. A maioria das recidivas (31 dos 32 casos) ocorreu em pacientes com LC tratados com $15 \mathrm{mg} \mathrm{Sb} b^{5+} / \mathrm{kg} /$ dia. Na investigação de critérios de cura, a reação intradérmica negativa foi o único fator associado a um risco três vezes maior de recidiva. Um aumento da dose ou do tempo de tratamento talvez melhore o prognóstico nestes pacientes.

Palavras-chaves: Leishmaniose tegumentar. Clínica. Propedêutica. Tratamento. Critérios de cura.

Abstract This study investigated clinical, laboratorial, therapeutic and prognostic aspects of American cutaneous leishmaniasis in Belo Horizonte in 358 patients with cutaneous leishmaniasis (CL) and 25 with mucocutaneous leishmaniasis (MCL). Compared to CL patients, the MCL patients reported longer duration of disease and higher frequency of other diseases, suggesting that debilitation caused by leishmaniasis or other conditions might contribute to activation and/or mucous dissemination of the parasite. The sensitivity of skin test, indirect immunofluorescence reactions and direct detection of parasites was 78.4, 79.3 and 68.3\%, respectively. The treatment with meglumine antimoniate presented $100 \%$ efficacy, but $59 \%$ patients had side-effects. During two years of follow-up, there were 32/318 relapses after successful treatment. Most relapses (31/32) were of CL patients treated with $15 \mathrm{mg} \mathrm{Sb} b^{5+} / \mathrm{kg} /$ day. The negative response to skin test was the only factor associated with a significant threefold increased risk of relapse. Higher dose or longer duration of treatment might improve the prognosis in these patients.

Key-words: Cutaneous leishmaniasis. Clinical and laboratory aspects. Antimonial treatment. Criteria of cure.

1. Faculdade de Medicina da Universidade Federal de Minas Gerais; 2. Centro de Pesquisas René Rachou da Fundação Oswaldo Cruz, Belo Horizonte, MG. Apoio Financeiro: PAPES/FIOCRUZ.

Endereço para correspondência: Dra. Valéria M. Azeredo Passos. Laboratório de Epidemiologia e Antropologia Médica/Centro de Pesquisas René Rachou. Av. Augusto de Lima 1715, 30190-002 Belo Horizonte, MG,

Fax: 5531 295-3115

e-mail:vpassos@cpqrr.fiocruz.br 
A leishmaniose tegumentar americana (LTA) foi uma parasitose de grande impacto médico e social no Brasil nas primeiras décadas deste século, quando a colonização agrícola colocava os trabalhadores expostos aos vetores do parasito em ambientes naturais. Na década de 40, afirmou-se que a progressão da derrubada de matas e a diminuição das possibilidades de criação de insetos transmissores, aliada à imunidade adquirida pela infecção, resultariam na diminuição ou mesmo desaparecimento da moléstia ${ }^{23}$. Entretanto, o que se observa é o recrudescimento da LTA, que passa a ter características de transmissão domiciliar e periurbana ${ }^{14}$. Casos autóctones de LTA têm sido registrados na Região Metropolitana de Belo Horizonte (RMBH) desde $1987^{21}$, sendo a Leishmania (Viannia) braziliensis a espécie predominantemente responsável pela infecção nesta região ${ }^{22}$.

A LTA ocorre, via de regra, em áreas com precárias condições sócio-econômicas ${ }^{14}$. Entretanto, existem poucos trabalhos avaliando o papel destes fatores na distribuição da infecção e de sua morbidade na população afetada. Conhecer a população com LTA na nossa região pode contribuir para o estabelecimento de medidas eficazes de controle da doença.
O tratamento da LTA apresenta duas limitações, a toxicidade das drogas disponíveis e a ausência de um critério de cura objetivo. Os antimoniais pentavalentes, apesar de sabidamente tóxicos, são as drogas de primeira linha no tratamento há mais de 40 anos $^{12}$. O controle de cura clínico é insatisfatório, pois são documentadas recidivas das lesões mesmo após tratamento e cicatrização completa da lesão inicial. O critério de cura parasitológico é pouco eficaz, dada a baixa sensibilidade da pesquisa do parasito mesmo antes do tratamento ${ }^{29}$. A presença de anticorpos anti-Leishmania após tratamento poderia significar persistência do parasitismo e ser fator preditivo para a recidiva da $\mathrm{LTA}^{25}$, sendo que a diminuição dos títulos da RIFI durante e após tratamento já foi sugerida como controle de cura ${ }^{4} 16$

O presente trabalho aborda a LTA a partir da descrição e análise dos fatores sócio-epidemiológicos, da forma de apresentação clínica, da resposta aos exames propedêuticos e da resposta à terapêutica antimonial. Além disso, este estudo utiliza a análise prospectiva para a investigação de fatores associados à recidiva da LTA após tratamento antimonial.

\section{MATERIAL E MÉTODOS}

Entre 1989 e 1995, foram atendidos 383 pacientes com LTA no ambulatório de leishmanioses do Centro de Pesquisas René Rachou-FIOCRUZ. Este ambulatório se localiza na cidade de Belo Horizonte e é referência de diagnóstico e tratamento para a $\mathrm{RMBH}$, composta de 18 municípios ao redor da capital, localizada na Zona Metalúrgica e Campo das Vertentes, na região central do Estado de Minas Gerais.

Este estudo possui dois componentes: 1) uma série temporal de casos desenvolvida para descrever as características sócio-epidemiológicas, clínicas e laboratoriais da LTA e 2) um estudo prospectivo desenvolvido para verificar as variáveis preditoras da recidiva da LTA após tratamento.

Após consentimento informado por escrito, foram incluídos os pacientes que apresentaram, além da suspeita clínica, pelo menos um exame complementar positivo: reação intradérmica de Montenegro (IDRM), reação de imunofluorescência indireta (RIFI) e/ou pesquisa do parasito ${ }^{7}$. Todos responderam a um protocolo pré definido com variáveis sócio-epidemiológicas (idade, gênero, cor, naturalidade, procedência, escolaridade, renda, ocupação e tipo de moradia) e clínicas (modalidade e duração da LTA; tipo, número e local das lesões, casos familiares de LTA, doenças pregressas e doenças simultâneas). A LTA foi dividida em LC e LM ${ }^{14}$, as lesões cutâneas foram localizadas por segmento corpóreo e classificadas por aspecto dermatológico ${ }^{8}$. As lesões mucosas foram investigadas com espéculo nasal e por laringoscopia indireta. Os pacientes com exame alterado eram enviados ao especialista para confirmação do diagnóstico, da cicatrização após tratamento e da suspeita de recidiva.
Diagnóstico laboratorial. A IDRM foi feita nos 383 pacientes, inoculando-se $0,1 \mathrm{ml}$ de antígeno feito de promastigotas mortas de L. (L.) amazonensis e padronizado em $40 \mathrm{mgN} / \mathrm{ml}$ na face anterior do antebraço ${ }^{15}$. A reação foi considerada positiva quando a área da enduração cutânea local era maior que $5 \mathrm{~mm}$ após 48 ou $72 \mathrm{~h}^{13}$. A RIFI para IgG anti-Leishmania foi feita em 334 pacientes que ingressaram no estudo a partir de 1990. $\mathrm{O}$ antígeno foi obtido de promastigotas cepa MHOM/BR/ $60 / \mathrm{BH} 6$, das espécie de $L$. (L.) mexicana/L. amazonensis a partir da classificação taxonômica de $1987^{14}$. Utilizaramse como controles negativos soros do pessoal do laboratório, sem história de lesão cutânea e com IDRM negativa. A reação foi considerada positiva a partir da segunda diluição de soro utilizada (titulação $\geq 1: 80)^{13}$. Um pequeno fragmento de tecido foi retirado da borda da lesão cutânea de 139 pacientes, em condições estéreis. O parasito foi visualizado através da impressão por aposição do tecido em lâmina de vidro e coloração pelo Giemsa.

Tratamento dos pacientes. Todos os pacientes foram tratados com antimoniato de meglumina (Glucantime ${ }^{\mathrm{R}}$ ), via intramuscular, na dose de $15 \mathrm{mg} / \mathrm{Sb}^{5+} / \mathrm{kg} /$ dia para LC e $20 \mathrm{mg} / \mathrm{Sb}^{5+} / \mathrm{kg} /$ dia para $\mathrm{LM}^{6}$, por ciclos de dez dias intercalados com 10 dias de repouso, em no mínimo 20 dias para LC e 30 dias para LM. A medicação era fornecida para dez dias e o paciente era instruído a retornar após 10 dias, a não usar bebidas alcóolicas e a interromper o tratamento e procurar assistência médica em caso de taquicardia ou oligúria. O controle eletrocardiográfico foi feito nos pacientes com idade $\geq 40$ anos. $O$ tratamento só foi interrompido após cicatrização completa da lesão ulcerada. Foram investigadas a observância da prescrição 
e a presença de efeitos colaterais após cada ciclo de tratamento.

Estudo prospectivo. Para efeito da análise, a entrada do paciente foi definida como a data do final do tratamento. Os pacientes incluídos foram avaliados clinicamente antes, durante e dez dias após o último ciclo de tratamento e em retornos semestrais por dois anos. A RIFI foi feita antes e dez dias após o tratamento. A contagem de tempo ocorreu: a) ao fim de dois anos de seguimento; b) na data da recidiva; c) na data da última consulta, para os pacientes que não retornaram a todos os controles semestrais ou d) na data da última consulta antes do fim do estudo em 30/6/1996. Foi considerada como recidiva, após tratamento e cicatrização total, a lesão cutânea em atividade no mesmo local da lesão cutânea anterior; a lesão mucosa nova em paciente com cicatriz de lesão cutânea anterior ou a lesão mucosa no mesmo local da lesão mucosa anterior. Ao final do estudo, foi fornecido relatório com instruções de retorno se houvesse suspeita de recidiva.

Análise estatística. Os dados foram analisados usando o pacote estatístico EGRET ${ }^{27}$. A investigação das variáveis associadas às manifestações clínicas da LTA foi feita através de regressão logística múltipla, calculando-se a razão de chance ( $\mathrm{RC}$ ) e o intervalo de confiança ao nível de 95\% (IC-95\%). Todas as variáveis associadas significativamente à forma de LTA estudada $(p<0,05) e$ aquelas definidas a prioricomo potenciais fatores de confusão foram incluídas na análise multivariada. As variáveis associadas à recidiva após tratamento foram examinadas utilizando-se as curvas de probabilidade Kaplan-Meyer. O modelo de risco proporcional de Cox foi usado para determinar o efeito independente das variáveis associadas à recidiva na análise univariada na predição da recidiva.

\section{RESULTADOS}

Participaram do estudo descritivo 250 (65,3\%) homens e $133(34,7 \%)$ mulheres, 216 (56,4\%) não-brancos e $167(43,6 \%)$ brancos, com idade entre 6 meses e 80 anos (mediana $=32$ anos). Cento e dez (28,7\%) pacientes residiam em Belo Horizonte, 151 (39,4\%) em outras cidades da $\mathrm{RMBH}, 116$ (30,3\%) em outras cidades de MG e 4 em outros Estados. Quanto à escolaridade, 75 (19,6\%) pacientes não foram à escola, $190(49,6 \%)$ freqüentaram até 4 anos e $118(30,8 \%)$ por mais de 4 anos. Cinqüenta e nove (15,4\%) pacientes recebiam menos de um salário mínimo (SM), 191 (49,9\%) de um a dois e 120 (31,3\%) 3 ou mais SM. Treze $(3,4 \%)$ pacientes estavam aposentados e 281 (73,4\%) exerciam atividades nãoagrícolas: $59(15,4 \%)$ pacientes eram menores, 39 (10,2\%) donas de casa, $28(7,3 \%)$ domésticas, $20(5,2 \%)$ serventes de pedreiro, 16 (4,2\%) comerciantes, 11 (2,9\%) pedreiros e $108(28,1 \%$ ) tinham outras ocupações. Apenas 89 (23,2\%) pacientes exerciam funções agrícolas: $78(20,4 \%)$ eram lavradores, sete $(1,8 \%)$ vaqueiros e quatro $(1 \%)$ lenhadores.

Apresentação clínica. A LC ocorreu em 358 (93,5\%) e a LM em $25(6,5 \%)$ pacientes. Dois pacientes apresentaram perfuração do septo nasal e um perfuração do palato. A lesão cutânea ocorreu em 365 pacientes, sendo do tipo úlcera franca em 250 (68,3\%), úlcera crostosa em 60 (16,4\%), cicatrização espontânea parcial em 22 (6\%), lesões vegetantes em 22 (6\%), pápulas em 7 (1,9\%) e lesões múltiplas de aspecto linfangítico ou esporotricóide em $5(1,4 \%)$ pacientes. Sete pacientes $(1,8 \%)$ apresentaram a forma LM de origem indeterminada, isto é, sem evidência de cicatriz ou lesão cutânea em atividade. As lesões cutâneas ocorreram em ordem decrescente em membros inferiores, superiores, cabeça, tronco, abdome e genitália. Além da LTA, 21 pacientes apresentavam infecção de vias aéreas superiores, quatro tinham pneumonia comunitária e um varicela.

$\mathrm{Na}$ análise univariada, a LM, quando comparada à LC, esteve significativamente associada à criação de animais no peridomicílio ( $\mathrm{RC}=2,94, \mathrm{IC}-1,29-6,71)$, à ausência de instalação sanitária $(R C=3,64$, IC-1,62$8,20)$, à ausência de água encanada $(R C=2,43$, IC$1,09-5,42)$ no domicílio, ao tempo de evolução da doença $\left(\chi_{\text {1tendência }}^{2}=93,58, p<0,001\right)$ e à presença de outra doença simultânea ( $R C=3,91, I C-1,12-14,07)$. O aumento da escolaridade e da renda estiveram inversamente associados com a ocorrência de LM $\left(\chi^{2}{ }_{1 \text { tendência }}=4,01\right.$, $p=0,045$ e $\left.\chi_{\text {tendência }}^{2}=8,93, p=0,003\right)$. A associação da LM com a renda e os indicadores de condições de moradia desapareceu no modelo multivariado, quando foi feito o ajuste por escolaridade e tempo de duração da LTA. As associações que se mantiveram estatisticamente significativas foram o tempo de evolução da doença e presença de doenças simultâneas (Tabela 1).

Diagnóstico laboratorial. A leitura da IDRM foi feita após 48h em 329 (85,9\%) e após $72 \mathrm{~h}$ em 36 (9,4\%) casos. Dezoito $(4,7 \%)$ pacientes não retornaram para a leitura. A reação foi positiva em 286 (78,4\%) dos 365 pacientes. Em 22 casos positivos, foi observada necrose no local de inoculação do antígeno. Esta reação foi mais freqüente nos pacientes com LM comparados aos pacientes com LC $\left(\chi_{1}^{2}=5,92, p<0,001\right)$. A RIFI foi positiva em $265(79,3 \%)$ dos 334 pacientes: $245(78,3 \%)$ das 313 reações positivas na LC e $20(95,2 \%)$ dos 21 pacientes na LM. A pesquisa do parasito foi positiva em 95 (68,3\%) dos 139 dos exames realizados.

Tratamento dos pacientes. A cicatrização completa das lesões ocorreu em 377 (98,4\%) dos 383 pacientes. Seis $(1,6 \%)$ pacientes não retornaram para o controle. 0 tempo de tratamento variou de 20 a 90 dias (mediana $=$ 20 dias). Doze (3\%) pacientes, onze com LC e um com LM, fizeram tratamento por menos de 20 dias, devido à melhora clínica e recusa em continuar a medicação. Quinze (60\%) pacientes com LM foram tratados por 30 dias, 5 (20\%) por 40 dias, $3(12 \%)$ por 50 dias e 1 (4\%) por setenta dias. Vinte e sete $(7 \%)$ pacientes tiveram intervalo maior que dez dias entre os ciclos. 
Tabela 1 - Fatores significativamente associados à apresentação da leishmaniose na forma mucosa na análise multivariada.

\begin{tabular}{lccc}
\hline \multirow{2}{*}{$\begin{array}{l}\text { Aspectos } \\
\text { clínicos }\end{array}$} & \multicolumn{2}{c}{ Leishmaniose } & $R^{*}$ \\
\cline { 2 - 3 } & $\begin{array}{c}\text { mucosa } \\
\mathrm{n}=25\end{array}$ & $\begin{array}{c}\text { cutânea } \\
\mathrm{n}=358\end{array}$ & $(\mathrm{IC}-95 \%)$ \\
\hline Duração da lesão & 2 & 152 & 1,00 \\
$<3 \mathrm{~m}$ & 3 & 139 & $1,51(0,3-9,1)$ \\
$3-5 \mathrm{~m}$ & 4 & 47 & $6,02(1,1-33,5)$ \\
$6-11 \mathrm{~m}$ & 16 & 20 & $35,6(7,7-165,0)$ \\
$\geq 12 \mathrm{~m}$ & $\chi_{1}^{2}=41,15$ & $p<0,01$ & \\
Doença simultânea & 19 & 331 & 1,00 \\
Não & 6 & 27 & $3,87(1,1-11,2)$ \\
Sim & & & \\
\hline
\end{tabular}

* Participaram do modelo inicial as variáveis : escolaridade, renda e ocupação.

$\mathrm{RC}=$ Razão de chance ajustada por escolaridade e variáveis listadas na tabela

IC = Intervalo de confiança

$\chi_{1}^{2}=$ qui-quadrado de tendência linear

Duzentos e vinte e cinco pacientes $(58,7 \%)$ queixaram algum efeito colateral: artralgia(10,2\%), hiporexia e náuseas $(9,2 \%)$, mialgia $(8,4 \%)$, dor no local da injeção $(3,9 \%)$, exantema máculo-papular extenso $(3,4 \%)$ e outras (cefaléia, tonteira e herpes zoster). As queixas foram mais comuns entre os adultos ( $\geq 18$ anos) $(\mathrm{RC}=3,55, \mathrm{IC} 95 \%-2,06-6,15)$. Todas as queixas foram tratadas sintomaticamente e não implicaram interrupção da droga. Não foi observada arritmia grave ou caso de morte súbita.

Preditores de recidivas após tratamento da LTA. Participaram do estudo prospectivo 318 pacientes, já que $65(16,9 \%)$ dos 383 pacientes não retornaram para o seguimento. Não houve diferenças significativas para as características estudadas entre os pacientes que permaneceram no estudo e as perdas para o seguimento. A recidiva ocorreu em 31 (10,3\%) dos 300 casos de LC e $1(5,5 \%)$ dos 18 casos de LM. Estes pacientes eram 24 homens e oito mulheres com idade entre um e 64 anos (média = 32,54 $\pm 17,29$ ), 21 não-brancos e 11 brancos. Quatro pacientes tinham renda inferior a um salário mínimo (SM), 16 de 1-2 SM e oito tinham renda $\geq 3 S M$. Vinte e nove casos de LC tiveram recidiva no mesmo local da lesão anterior, sendo que dois pacientes apresentaram também lesão mucosa nova. O paciente com recidiva da LM apresentou lesão em atividade no mesmo local da lesão mucosa anterior. Dois pacientes com recidiva tinham interrompido o tratamento com $10 \mathrm{e}$ 12 dias, devido à melhora clínica. Um paciente teve intervalo de 19 dias entre dois ciclos. Todos os pacientes receberam outro tratamento antimonial, na mesma dose anterior, com cicatrização total das lesões. Seis pacientes apresentaram um segundo episódio de recidiva. Por dificuldade de utilização de outras drogas, eles receberam novo tratamento com antimonial, por 30 dias seguidos, com cicatrizacãa das lesões e ausência de recidiva durante os 12 meses posteriores de seguimento.

O risco acumulado em dois anos para a ocorrência de recidiva foi igual a 10,5\%, com 30 casos em até $12 \mathrm{~m}$ após tratamento (Tabela 2). A curva de probabilidade foi proporcional ao tempo e permitiu a análise usando

Tabela 2 - Probabilidade de recidiva em pacientes com LTA segundo o tempo de seguimento.

\begin{tabular}{lccc}
\hline $\begin{array}{l}\text { Seguimento } \\
\text { (meses) }\end{array}$ & $\begin{array}{c}\text { Pessoas } \\
\text { sob risco }\end{array}$ & $\begin{array}{c}\text { Número } \\
\text { de recidivas }\end{array}$ & $\begin{array}{c}\text { Probabilidade de } \\
\text { recidiva }(\text { IC 95\%) }\end{array}$ \\
\hline 1 & 318 & 1 & $0,3(0,0-2,0)$ \\
2 & 317 & 11 & $3,8(2,0-6,0)$ \\
3 & 306 & 7 & $6,0(4,0-9,0)$ \\
4 & 299 & 3 & $6,9(5,0-10,0)$ \\
5 & 296 & 1 & $7,2(5,0-11,0)$ \\
6 & 295 & 3 & $8,2(6,0-13,0)$ \\
8 & 259 & 2 & $8,9(6,0-13,0)$ \\
9 & 255 & 1 & $9,2(7,0-13,0)$ \\
12 & 248 & 1 & $9,6(7,0-14,0)$ \\
13 & 225 & 1 & $10,0(7,0-14,0)$ \\
23 & 179 & 1 & $10,5(7,0-14,0)$ \\
\hline
\end{tabular}

IC = Intervalo de confiança 
o modelo de risco proporcional de Cox. Não houve associação entre a recidiva e aspectos epidemiológicos ou clínicos da LTA. A única associação evidenciada foi entre a resposta à IDRM e a recidiva, com a resposta negativa à IDRM aumentando em mais de três vezes o risco de recidiva. A média geométrica da razão inversa dos títulos da RIFI foi igual a 89,1 e 38,9 antes e após tratamento, respectivamente; com uma diminuição significativa dos títulos após o tratamento $(p<0,001)$. Entretanto, a resposta à RIFI, tanto antes como após tratamento, não foi associada ao risco de recidiva (Tabelas 3 e 4$)$.

\begin{tabular}{|c|c|c|c|c|}
\hline \multirow{2}{*}{$\begin{array}{l}\text { Aspectos } \\
\text { sócio-epidemiológicos }\end{array}$} & \multirow{2}{*}{$\begin{array}{c}\text { Total de } \\
\text { Pacientes } \\
(\mathrm{n}=318)\end{array}$} & \multicolumn{2}{|c|}{ Pacientes } & \multirow{2}{*}{$\begin{array}{l}\text { Razão de risco } \\
\text { (IC -95\%) }\end{array}$} \\
\hline & & $\begin{array}{c}\text { com recidiva } \\
(\mathrm{n}=32)\end{array}$ & $\begin{array}{c}\text { sem recidiva } \\
(\mathrm{n}=286)\end{array}$ & \\
\hline \multicolumn{5}{|l|}{ Gênero } \\
\hline Masculino & 207 (65\%) & 24 & 203 & 1,00 \\
\hline Feminino & $111(35 \%)$ & 8 & 103 & $0,59(0,27-1,32)$ \\
\hline \multicolumn{5}{|l|}{ Idade } \\
\hline $0-9$ anos & 29 ( $9 \%)$ & 3 & 26 & 1,00 \\
\hline $10-50$ anos & $212(67 \%)$ & 24 & 188 & $1,13(0,34-3,75)$ \\
\hline$\geq 50$ anos & 77 (24\%) & 5 & 72 & $0,64(0,15-2,69)$ \\
\hline \multicolumn{5}{|l|}{ Cor } \\
\hline Não-branca & $180(57 \%)$ & 21 & 159 & 1,00 \\
\hline Branca & $138(43 \%)$ & 11 & 127 & $0,66(0,32-1,38)$ \\
\hline \multicolumn{5}{|l|}{ Educação } \\
\hline 0 & 59 (18\%) & 8 & 51 & 1,00 \\
\hline 1-4 anos & $158(50 \%)$ & 13 & 145 & $0,58(0,24-1,41)$ \\
\hline$\geq 4$ anos & $101(32 \%)$ & 11 & 90 & $0,80(0,32-2,00)$ \\
\hline \multicolumn{5}{|l|}{ Renda } \\
\hline$<1 \mathrm{SM}$ & $45(15 \%)$ & 4 & 41 & 1,00 \\
\hline $1-2 \mathrm{SM}$ & $158(51 \%)$ & 17 & 141 & $1,22(0,41-3,64)$ \\
\hline$\geq 3 \mathrm{SM}$ & $103(34 \%)$ & 7 & 96 & $0,77(0,23-2,65)$ \\
\hline $\operatorname{lgn}$ & 12 & 4 & 8 & \\
\hline \multicolumn{5}{|l|}{ Ocupação } \\
\hline Agrícola & 71 (22\%) & 5 & 66 & 1,00 \\
\hline Não-agrícola & $247(78 \%)$ & 27 & 220 & $1,63(0,65-4,12)$ \\
\hline
\end{tabular}

IC- Intervalo de confiança

Tabela 4 - Risco de recidiva para pacientes com LTA segundo aspectos clínico-laboratoriais (estudo prospectivo com 318 pacientes).

\begin{tabular}{|c|c|c|c|c|}
\hline \multirow{2}{*}{$\begin{array}{l}\text { Aspectos clínicos } \\
\text { e laboratoriais }\end{array}$} & \multirow{2}{*}{$\begin{array}{c}\text { Total de } \\
\text { Pacientes } \\
(\mathrm{n}=318)\end{array}$} & \multicolumn{2}{|c|}{ Pacientes } & \multirow{2}{*}{$\begin{array}{l}\text { Razão de risco } \\
\text { (IC -95\%) }\end{array}$} \\
\hline & & $\begin{array}{c}\text { com recidiva } \\
(\mathrm{n}=32)\end{array}$ & $\begin{array}{c}\text { sem recidiva } \\
(\mathrm{n}=286)\end{array}$ & \\
\hline \multicolumn{5}{|c|}{ IDRM ao diagnóstico } \\
\hline Positiva & $240(79 \%)$ & 17 & 223 & 1,00 \\
\hline Negativa & $63(21 \%)$ & 14 & 49 & $3,44(1,70-6,99)$ \\
\hline $\lg n$ & 15 & 1 & 14 & \\
\hline \multicolumn{5}{|l|}{ RIFI ao diagnóstico } \\
\hline Positiva & $220(79 \%)$ & 24 & 196 & 1,00 \\
\hline Negativa & $60(21 \%)$ & 3 & 57 & $2,19(0,66-7,29)$ \\
\hline $\operatorname{lgn}$ & 38 & 5 & 33 & \\
\hline \multicolumn{5}{|c|}{ Redução da RIFI após tratamento } \\
\hline Não & $131(50 \%)$ & 14 & 117 & 1.00 \\
\hline Sim & $132(50 \%)$ & 13 & 119 & $0,92(0,43-1,95)$ \\
\hline $\lg n$ & 55 & 5 & 50 & \\
\hline \multicolumn{5}{|l|}{ Tratamento } \\
\hline Suficiente & $311(98 \%)$ & 30 & 281 & 1,00 \\
\hline Insuficiente & $7(2 \%)$ & 2 & 5 & $2,19(0,26-18,21)$ \\
\hline \multicolumn{5}{|l|}{ Leishmaniose } \\
\hline Cutânea & $300(94 \%)$ & 31 & 269 & 1,00 \\
\hline Mucosa & $18(6 \%)$ & 1 & 17 & $0,52(0,01-3,86)$ \\
\hline \multicolumn{5}{|c|}{ Leishmaniose cutânea } \\
\hline Lesão única & $218(69 \%)$ & 20 & 198 & 1,00 \\
\hline Lesão múltipla & $100(31 \%)$ & 12 & 88 & $1,41(0,68-2,88)$ \\
\hline \multicolumn{5}{|l|}{ Duração da lesão } \\
\hline$<3$ meses & $131(41 \%)$ & 19 & 112 & 1,00 \\
\hline 3-5 meses & $118(37 \%)$ & 6 & 112 & $0,34(0,13-0,84)$ \\
\hline 6-11 meses & $40(13 \%)$ & 5 & 35 & $0,84(0,31-2,25)$ \\
\hline$\geq 12$ meses & 29 ( $9 \%)$ & 2 & 27 & $0,45(0,10-2,92)$ \\
\hline \multicolumn{5}{|l|}{ Doença simultânea } \\
\hline Não & 292 (92\%) & 29 & 263 & 1,00 \\
\hline Sim & $26(8 \%)$ & 3 & 23 & $1,18(0,36-3,87)$ \\
\hline
\end{tabular}




\section{DISCUSSÃO}

A distribuição por gênero e idade sugere a coexistência de dois modelos de transmissão da LTA. O maior atendimento de homens e de adultos sugere transmissão extradomiciliar em população economicamente ativa ${ }^{18}$, enquanto que o atendimento de mulheres, crianças e pessoas com ocupações não agrícolas sugere a transmissão intra e/ou peridomiciliar ${ }^{19}{ }^{21}$. A RMBH foi o local provável de infecção mais relatado, refletindo uma nova situação epidemiológica, com a expansão da LTA a regiões até então indenes ${ }^{21}$. A baixa escolaridade e renda e o predomínio de ocupações pouco qualificadas reforçam que a LTA, assim como a maioria das doenças infecto-parasitárias, atinge principalmente as populações mais carentes. O reduzido percentual de ocupações agrícolas reflete o atendimento em área urbana com casos autóctones ${ }^{19}$.

O predomínio em áreas expostas do corpo e do aspecto ulcerado correspondem à descrição mais freqüente das lesões cutâneas por LTA $^{8}$. A ocorrência de lesões múltiplas em um terço dos pacientes: pode ser devida a várias picadas do vetor infectado, explicando os casos de lesões mais distantes entre si. A disseminação linfática e/ou hematogênica em trajeto linear no mesmo segmento corpóreo determinou lesões de aspecto espotricóide ou linfangítico. Somente após terapia específica, foi observada a cicatrização completa dos 20 casos de cicatrização parcial, ou o fim do eritema da borda das lesões nos dois casos de lesões já cicatrizadas, sugerindo a persistência do parasito nestas lesões.

Apesar de menos freqüente, a LM é geralmente mais grave, podendo deixar seqüelas. A LM está principalmente associada à infecção por L. braziliensis ${ }^{14}$ e sua prevalência em áreas endêmicas depende da virulência do parasito, da imunocompetência do hospedeiro e da assistência médica. Sua freqüência vem diminuindo em áreas endêmicas, não excedendo a $3 \%$, provavelmente devido ao diagnóstico e tratamento precoces ${ }^{14}$. Os $6,5 \%$ de casos de LM deste estudo podem se dever à instalação mais recente da LTA na $\mathrm{RMBH}$. A associação entre a LM e características das lesões cutâneas ${ }^{10}$ descritas em análise univariada, não foram encontradas neste estudo. O desaparecimento da associação entre a LM e piores condições sócio-econômicas, após o ajuste por tempo da lesão, indica que o tempo de evolução é o fator biológico que determina, em última análise, a disseminação do parasito. Entretanto, as piores condições de renda e escolaridade estão sem dúvida, na raiz deste tempo prolongado da doença em paciente que não tem acesso, ou não procura assistência médica em tempo hábil. $O$ maior tempo de evolução e a maior freqüência de outras doenças simultâneas na LM, sugerem que a debilitação pela própria doença e/ou outras doenças podem contribuir para a ativação e/ou disseminação mucosa do parasito. Entretanto, não é possível definir com certeza se estes fatores foram causa ou conseqüência da LM.
A sensibilidade da IDRM verificada neste estudo foi menor que a relatada na padronização da reação (96\%) considerando como reação positiva a presença de vesícula ou área de enduração perceptível ao tato ${ }^{15}$. Neste estudo, só foram consideradas positivas as endurações com diâmetro $>5 \mathrm{~mm}$, o que pode ter contribuído para a menor sensibilidade. Em estudo com o mesmo antígeno e critério de positividade deste estudo, a sensibilidade foi igual a $83,6 \%{ }^{28}$. A menor sensibilidade pode refletir a diferença entre os valores encontrados no laboratório e nos serviços de saúde, já que o antígeno deve ser mantido a $4^{\circ} \mathrm{C}$ e diferenças na temperatura podem interferir com a sua qualidade. A maior freqüência de necrose no local de inoculação do antígeno, nos pacientes com LM, pode se dever a uma resposta imune celular exacerbada pela maior duração da doença e a estimulação prolongada de linfócitos, na ausência de uma modulação eficiente ${ }^{6}$.

A comparação da sensibilidade da RIFI com os resultados obtidos em outras áreas endêmicas foi semelhante à da literatura ${ }^{516}$, apesar das diferenças nos antígenos utilizados.

A boa eficácia terapêutica observada já foi relatada com as doses utilizadas neste estudo ${ }^{28}$ ou com dose menor ${ }^{20}$. Esta eficácia pode estar relacionada a características do parasito ${ }^{20}$ e/ou ao pouco tempo de evolução $(<6 \mathrm{~m})$ das lesões na maioria $(75 \%)$ dos pacientes. Evidências de disseminação linfática dos parasitos antes do aparecimento da lesão primária ${ }^{3}$ reforçam a necessidade do tratamento precoce. Apesar da baixa escolaridade e renda da população atendida, o pequeno percentual de abandono de tratamento observado neste estudo (1,6\%) quando comparados ao percentual de $25 \%$ já relatado ${ }^{11}$ na $\mathrm{RMBH}$, pode ser atribuído aos esclarecimentos prestados e/ou ao fornecimento do medicamento e seringas suficientes para apenas 10 dias.

O tratamento antimonial por 20 dias seguidos tem sido recomendado na tentativa de diminuir as recidivas ${ }^{12}$. Entretanto, quando comparado ao tratamento em ciclos de dez dias, o tratamento por 20 dias seguidos apresentou maior freqüência de abandono e conseqüente maior necessidade de tratamento adicional, além de maior incidência de efeitos colaterais ${ }^{1}$.

Os efeitos colaterais observados coincidem com os já descritos ${ }^{8}{ }^{12}$. A maior incidência em adultos reflete provavelmente uma relação dose-efeito. Embora antimoniais em doses altas possam levar a arritmias e à morte súbita, as doses por nós utilizadas se revelaram seguras $^{24}$.

A freqüência de recidivas após tratamento foi semelhante à encontrada em outra área de LTA por $L$. braziliensis ${ }^{17}$. A ocorrência de mais de $90 \%$ das recidivas em até um ano reforça a sugestão de controle clínico por pelo menos um ano após tratamento ${ }^{29}$. 
A recidiva da LTA pode ser devida a uma infecção persistente ou à reinfecção ${ }^{26}$. Neste estudo, só foi considerada recidiva a lesão que ocorresse na cicatriz da lesão anterior ou então primariamente na mucosa, reforçando a hipótese de reativação de infecção nos nossos casos. Esta reativação se deve à falência do tratamento ou então, à hipótese que, pelo menos na leishmaniose por L. braziliensis, a cura clínica é alcançada sem a erradicação dos parasitos ${ }^{9}$. 0 tratamento com dose ou tempo insuficiente foi raro e não esteve relacionado à incidência de recidivas. Entretanto, o esquema de tratamento em ciclos de dez dias pode ter contribuído para a ocorrência de recidiva ${ }^{12}$. O risco de recidiva para os casos de LC e de LM foi igual. Dado o maior tempo de doença e a maior disseminação do parasita na LM, seria esperado um maior risco para recidiva nestes casos. A maior dose e maior tempo de uso do antimonial nos pacientes com LM devem ter influenciado na menor ocorrência de recidivas, reforçando a recomendação para uso de $20 \mathrm{mgSb}^{5+} / \mathrm{kg} /$ dia para todos os casos de LC por L. braziliensis ${ }^{122}$.

Nenhum aspecto clínico ou epidemiológico esteve associado à ocorrência de recidivas. A resposta de anticorpos medida pela RIFI não foi associada a este risco. A diminuição dos títulos após tratamento é, provavelmente, devida à menor estimulação antigênica parasitária. Nem a sorologia qualitativa nem a quantitativa estiveram associadas ao risco de recidiva, sugerindo que a resposta imune humoral tenha pouca ou nenhuma ação protetora na $\mathrm{LTA}^{2}$.

A IDRM negativa foi o único fator prognóstico para a incidência de recidivas até dois anos após o tratamento. A resposta imune celular é importante para a recuperação do paciente e para a resistência à re-infecção em todas as formas de leishmanioses. A IDRM, teste de hipersensibilidade cutânea tardia, que foi a primeira forma de avaliação da imunidade na LTA, se correlaciona com a proteção e é útil na avaliação prognóstica². Os pacientes com LC difusa apresentam IDRM geralmente negativa, se tornando positiva nos raros períodos de melhora da doença ${ }^{2}$. Nossos dados indicam que também nas modalidades cutânea e mucosa da LTA, a positividade do teste cutâneo, aferida quando do diagnóstico, indica proteção do indivíduo, com menor incidência de recidiva. Pacientes com LTA e IDRM negativa devem ser considerados de maior risco de recidiva das lesões e acompanhados com maior rigor após tratamento. Além disso, alterações na dose ou tempo de tratamento dos pacientes com IDRM negativa podem contribuir para a menor incidência de recidivas.

\section{AGRADECIMENTOS}

Os autores VMA Passos, MFF Lima-Costa, AU Krettli, AJ Romanha e AL Falcão agradecem ao CNPq pela bolsa de produtividade e AC Volpini agradece à FIOCRUZ pela bolsa de Pós-graduação.

\section{REFERÊNCIAS BIBLIOGRÁFICAS}

1. Azeredo-Coutinho RB, Mendonça SCF. Comparative study of two antimonial therapy schedules for treating cutaneous leishmaniasis. Memórias do Instituto Oswaldo Cruz 92(supl.I):223, 1997.

2. Barral A. Imuno-regulação na leishmaniose tegumentar. Revista da Sociedade Brasileira de Medicina Tropical 27(supl.4):346-348, 1994.

3. Barral A, Guerreiro J, Bonfim G, Correia D, Barral-Neto M, Carvalho EM. Lymphadenopathy as the first sign of human cutaneous infection by Leishmania braziliensis. American Journal of Tropical Medicine and Hygiene 53:256-259, 1995.

4. Chiari CA, Mayrink W, Magalhães PA. Reação de imunofluorescência indireta no controle do tratamento da leishmaniose tegumentar americana. Revista do Instituto de Medicina Tropical de São Paulo 15:298-303, 1973.

5. Cuba-Cuba C, Llanos-Cuentas EA, Barreto AC, Magalhães AV, Lago EL, Reed SG, Marsden PD. Human mucocutaneous leishmaniasis in Três Braços, Bahia-Brazil. An area of Leishmania braziliensis braziliensis transmission. I. Laboratory diagnosis. Revista da Sociedade Brasileira de Medicina Tropical 7:161-167, 1984.

6. Fundação Nacional de Saúde, Centro Nacional de Epidemiologia, Coordenação Nacional de Dermatologia Sanitária. Guia de controle da Leishmaniose Tegumentar Americana, Ministério da Saúde, Brasília, 1994.
7. Furtado TA. Critérios para o diagnóstico da leishmaniose tegumentar americana. Anais Brasileiros de Dermatologia 55:81$86,1980$.

8. Furtado TA. Leishmaniose Tegumentar Americana. In: MachadoPinto J. Doenças infecciosas com manifestações dermatológicas. Ed. Médica e Científica Ltda, p.319-328, 1994.

9. Guevara P, Ramirez JL, Rojas E, Scorza JV, González N, Añez $\mathrm{N}$. Leishmania braziliensis in blood 30 years after cure. The Lancet 341: 1341, 1993.

10. Llanos-Cuentas EA, Marsden PD, Lago EL, Barreto AC, CubaCuba C, Johnson WD. Human mucocutaneous leishmaniasis in Três Braços, Bahia-Brazil. An area of Leishmania braziliensis braziliensis transmission. II. Cutaneous disease. Presentation and evolution. Revista da Sociedade Brasileira de Medicina Tropical 17:169-177, 1984.

11. Lucciola GV, Passos VMA, Patrus AO. Mudança no padrão epidemiológico da leishmaniose tegumentar americana. Anais Brasileiros de Dermatologia 71:99-105, 1996.

12. Marsden PD. Novos conceitos sobre os antimoniais pentavalentes no tratamento da leishmaniose. Revista da Sociedade Brasileira de Medicina Tropical 16: 172-174, 1983.

13. Marzochi MCA, Coutinho SG, Sabrosa PC, Souza WJS. Reação de imunofluorescência indireta e intradermoreação para 
leishmaniose tegumentar americana em moradores na área de Jacarepaguá. Estudo comparativo dos resultados obtidos em 1974 e 1978. Revista do Instituto de Medicina Tropical de São Paulo 22:149-155, 1980.

14. Marzochi MCA, Marzochi KBF. Tegumentary and Visceral Leishmaniasis in Brazil- emerging Anthropozoonosis and possibilities for their control. Cadernos de Saúde Pública 10(supl.2):359-375, 1994.

15. Melo MN, Mayrink W, Costa CA, Magalhães PA, Dias M, Williams P, Araújo FG, Coelho M, Batista SM. Padronização do antígeno de Montenegro. Revista do Instituto de Medicina Tropical de São Paulo 6:171-176, 1977.

16. Mendonça SCF, Souza WJS, Nunes MP, Marzochi MCA, Coutinho SG. Indirect immunofluorescence test in New World leishmaniasis: serological and clinical relationship. Memórias do Instituto Oswaldo Cruz 83:347-355, 1988.

17. Netto EM, Marsden PD, Llanos-Cuentas EA, Costa JML, CubaCuba C, Barreto AC, Badaró R, Johnson WD. Long-term followup of patients with Leishmania (Viannia) braziliensis infection and treated with Glucantime®. Transactions of the Royal Society of Tropical Medicine and Hygiene 84:367-370, 1990.

18. Oliveira MRF, Macedo VO, Carvalho EM, Barral A, Marroti JG, Bittencout A, Abreu MVA, Orge MGO, Lessa HA, Marsden PD. Estudo evolutivo da leishmaniose mucosa ( 7 a 17anos) causada por Leishmania (Viannia) braziliensis em Três Braços, Bahia Revista da Sociedade Brasileira de Medicina Tropical 28:325332, 1995.

19. Oliveira-Neto MP, Pirmez C, Rangel R, Schubach A, Grimaldi JrG. An outbreak of american cutaneous leishmaniasis (Leishmania braziliensis braziliensis) in a periurban area of city, Brazil: clinical and epidemiological studies. Memórias do Instituto Oswaldo Cruz 83:427-435, 1988.

20. Oliveira-Neto MP, Schubach A, Mattos M, Gonçalves-Costa SC, Pirmez C. A low dose antimony treatment in 159 patients with American Cutaneous Leishmaniasis: extensive follow-up studies (up to 10 years). American Journal of Tropical Medicine and Hygiene 57:651-655, 1997.
21. Passos VMA, Falcão AL, Marzochi MCA, Gontijo CMF, Dias ES Barbosa-Santos EGO, Guerra HL, Katz N. Epidemiological aspects of American Cutaneous Leishmaniasis in a periurban area of the Metropolitan Region of Belo Horizonte, Minas Gerais, Brazil. Memórias do Instituto Oswaldo Cruz 88:103-110, 1993.

22. Passos VMA, Fernandes O, Lacerda PAF, Volpini AC, Gontijo CMF, Degrave W, Romanha AJ. Leishmania (Viannia) braziliensis is the predominant species infecting patients with American cutaneous leishmaniasis in the State of Minas Gerais, Southeast Brazil. Acta Tropica 72:251-258, 1999.

23. Pessoa SB, Barreto MP. Epidemiologia. In: Pessoa SB, Barreto MP (eds) Leishmaniose tegumentar americana. $2^{a}$ edição, p. 361 419, 1948

24. Ribeiro ALP, Drummond JB, Volpini AC, Andrade AC, Passos VM. Electrocardiographic changes during low-dose, short-term therapy of cutaneous leishmaniasis with the pentavalent antimonial meglumine. Brazilian Journal of Medical and Biological Research 32:297-301 1999.

25. Saravia NG, Valderrama L, Labrada M, Holguin AF, Navas C, Palma G, Weigle KA. The relationship of Leishmania braziliensis subecies and immune response to disease expression in New World Leishmaniasis. The Journal of Infectious Diseases 159:725735,1989

26. Saravia NG, Weigle K, Segura I, Giannini SH, Pacheco R, Labrada LA, Gonçalves A. Recurrent lesions in human Leishmania braziliensis infection- reactivation or reinfection? The Lancet 336:398-402, 1990.

27. United States, Statistics and Epidemiology Research to Egret: Upgrade document: changes from previous versions, 1990.

28. Wanke CFN, Birkenhauer MC, Maceira JMP, Silva FC, Perez M. Leishmaniose tegumentar: estudo retrospectivo de 65 casos. Anais Brasileiros de Dermatologia 66:49-54, 1991.

29. World Health Organization. Expert Committee on the Control of the Leishmaniases. Genebra:WHO 1990. 